\title{
Uso neoadjuvante do mesilato de imatinibe no tratamento de GIST retal volumoso: relato de caso
}

\author{
Neoadjuvant use of imatinib mesylate for treatment of \\ large rectal GIST: case report
}

\author{
PAULO ROCHA FRANÇA NETOํ, LEANDRO ALVES GOMES RAMOS ${ }^{2}$, LUCIANA COSTA SILVA 3 , \\ CRISTIANE KOIZIMI M. FERNANDES ${ }^{1}$, ANTÔNIO LACERDA-FILHO ${ }^{1}$
}

\begin{abstract}
${ }^{1}$ Clínica Coloproctológica do Hospital Felício Rocho. ${ }^{2}$ ONCOMED-BH - Clínica de Tratamento e Prevenção de Doenças Oncológicas. ${ }^{3}$ Clínica ECOAR - Medicina Diagnóstica - Belo Horizonte (MG), Brasil.
\end{abstract}

\begin{abstract}
NETO PRF, RAMOS LAG, SILVA LC, FERNANDES CKM, LACERDA-FILHO A. Uso neoadjuvante do mesilato de imatinibe no tratamento de GIST retal volumoso: relato de caso. Rev bras Coloproct, 2011;31(1): 89-93.

RESUMO: Tem sido relevante o papel das drogas que interferem na atividade tirosina-quinase dos receptores $c$-kit, no tratamento dos tumores derivados do estroma gastrintestinal (GISTs), sobretudo em tumores volumosos. Relata-se o caso de um paciente do sexo masculino, 56 anos, obeso, com quadro de peso retoanal associado a tenesmo e à sensação de evacuação incompleta. Foi diagnosticado volumoso GIST de reto inferior de localização posterior, visualizado por ressonância magnética e confirmado por estudo imunoistoquímico em punção-biópsia parassacral, guiada por tomografia. A impressão inicial foi de necessidade de amputação abdômino-perineal do reto, pois havia importante compressão do canal anal e do aparelho esfincteriano. Optou-se, então, por indicação de neoadjuvância com mesilato de imatinibe (Glivec ${ }^{\circledR}$ ) na tentativa de preservação esfincteriana. Após quatro meses de tratamento, apresentava, ao toque retal, redução significativa (cerca de $50 \%$ ) do volume da massa e em menor grau à ressonância magnética. Paciente foi submetido à excisão total do mesorreto e anastomose colo-anal manual, com ileostomia protetora. Evoluiu com necrose do cólon abaixado, tendo sido realizada ressecção do mesmo e colostomia terminal ilíaca. O paciente recusou a se submeter a uma nova tentativa de abaixamento colo-anal, tendo sido fechada a ileostomia e restabelecido trânsito pela colostomia ilíaca. No tratamento dos GISTs de reto muito volumosos ou irressecáveis, deve-se avaliar a indicação pré-operatória do imatinibe, uma vez que a cirurgia radical deve ser sempre indicada, a fim de minimizar a possibilidade de recorrência local.
\end{abstract}

Palavras-chave: câncer retal; neoplasias colorretais; cirurgia; antineoplásicos.

\section{INTRODUÇÃO}

Os tumores do estroma gastrintestinal ou gastrointestinal stromal tumor (GIST) são neoplasias mesenquimais que se originam da parede de vísceras ocas do trato gastrintestinal, podendo atingir o intestino grosso em cerca de $5 \%$ dos casos, principalmente o reto. A ressecção cirúrgica, dentro dos princípios oncológicos, é a base do tratamento destas neoplasias.
Por outro lado, tem sido relevante o papel das drogas que interferem na atividade tirosina-quinase dos receptores para fator de crescimento tumoral, como o mesilato de imatinibe. Tais drogas vêm sendo utilizadas no tratamento dos GISTs volumosos ou metastáticos, com resultados promissores. Ultimamente, têm surgido relatos de sua utilização também como tratamento neoadjuvante de tumores volumosos, no intuito de diminuir seu volume, facilitando a abordagem cirúrgica e

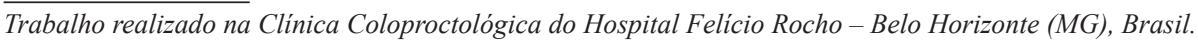

Conflito de interesse: nada a declarar.

Recebido em: 06/11/2009

Aprovado em: 14/04/2011 
diminuindo sua morbimortalidade. Especialmente nos GISTs de reto, essa prática pode ter grande relevância, já que pode favorecer a preservação do aparelho esfincteriano. $\mathrm{O}$ caso apresentado a seguir procura demonstrar os resultados desta abordagem terapêutica.

\section{RELATO DO CASO}

Paciente do sexo masculino, 56 anos, obeso, com história de peso reto-anal com cerca de um mês de evolução, associado a tenesmo e à sensação de evacuação incompleta. Trazia uma colonoscopia feita há um ano, realizada até o ceco, sem alterações. Portador de hipertensão arterial sistêmica e diabetes tipo II, associados à obesidade. Ao toque retal, ele apresentava massa retro-retal volumosa, comprimindo o canal anal posteriormente ao nível do anel anorretal, com mucosa lisa e intimamente aderida ao reto. Retossigmoidoscopia foi normal até $15 \mathrm{~cm}$. O paciente foi submetido à ressonância nuclear magnética de pelve, que mostrou volumosa lesão retro-retal contida dentro dos limites do mesorreto, heterogênea com áreas de necrose central, acometendo até o nível dos elevadores, sugestiva do GIST retal (Figura 1). Optou-se por realizar biópsia parassacral por agulha, guiada por tomografia, a qual à imunoistoquímica confirmou a presença de GIST retal (positividade para c-kit e CD 34).

Diante da indicação de amputação abdôminoperineal do reto de princípio, optou-se por tratamento neoadjuvante com mesilato de imatinibe aos cuidados da Oncologia clínica, a fim de obter-se possível diminuição da massa e preservação do aparelho esfincteriano. Foi utilizada a dose diária de $400 \mathrm{mg}$, durante quatro meses.

Após esse período, o paciente retornou para nova avaliação, quando apresentava, ao toque retal, redução significativa da massa (cerca de 50\%) e em menor grau à ressonância magnética de controle (Figura 2). Após a neoadjuvância, foi possível a preservação esfincteriana, sendo realizada excisão total do mesorreto (Figuras 3 e 4) e anastomose colo-anal manual com ileostomia protetora, tendo havido grande dificuldade técnica para a realização do abaixamento do cólon, devido à presença de mesocólon curto e espesso, com arcadas vasculares desfavoráveis.

No pós-operatório, o paciente evoluiu com isquemia do cólon abaixado, sendo que foi realizada sua

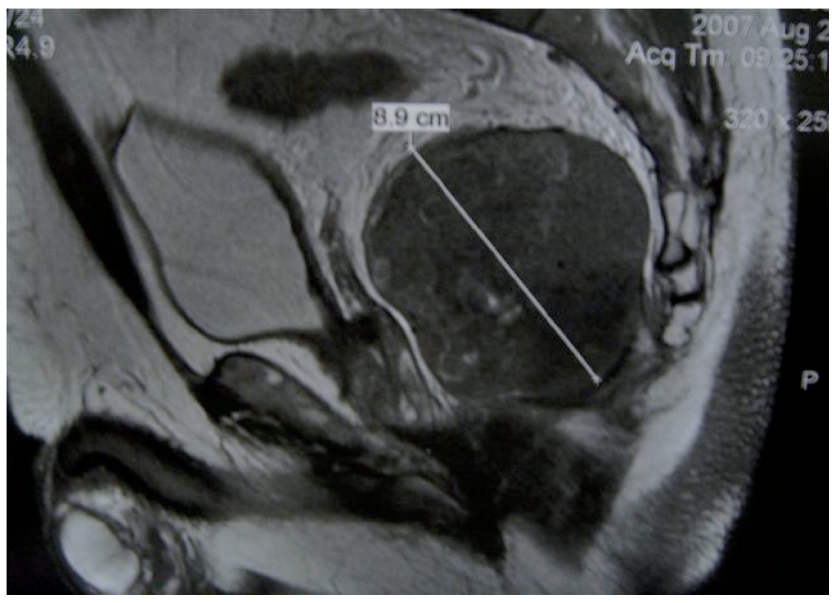

Figura 1. Imagem de ressonância nuclear magnética, mostrando lesão retro-retal volumosa.

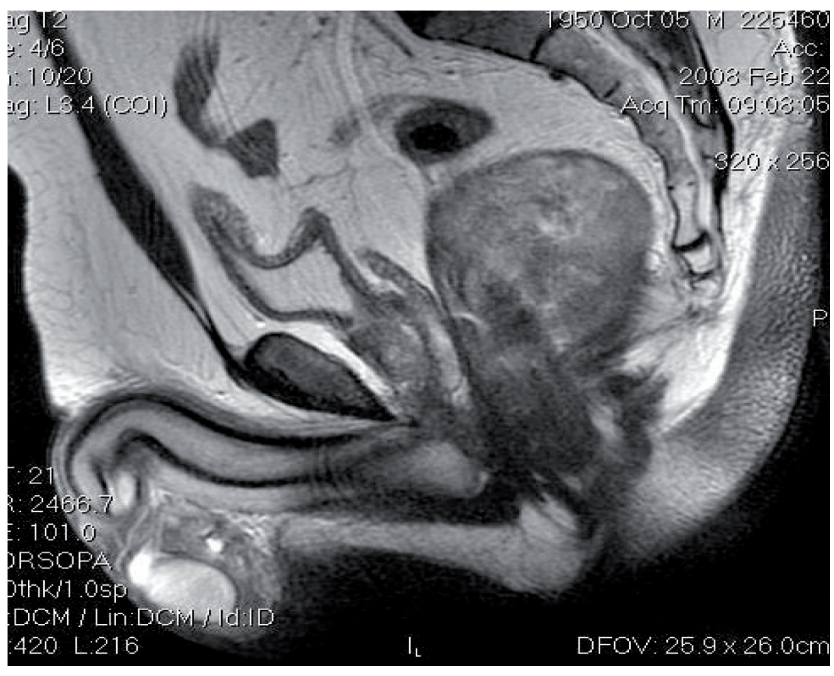

Figura 2. Imagem de ressonância nuclear magnética de GIST retal pós-neoadjuvância, com diminuição do tamanho do tumor.

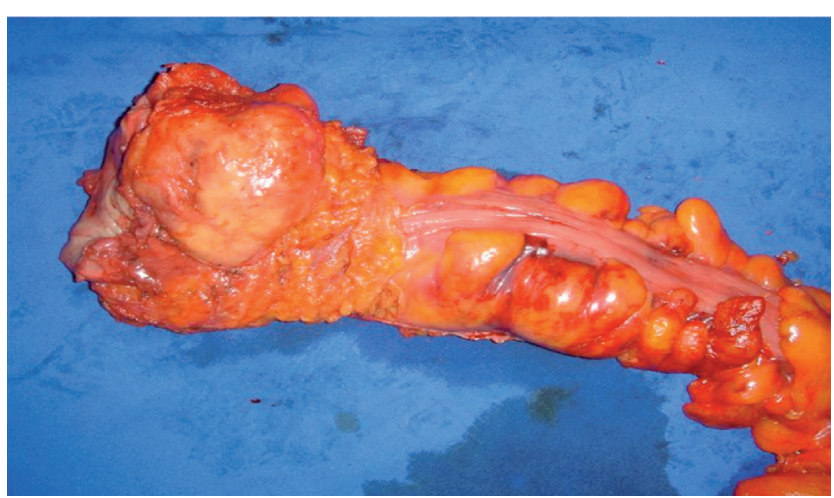

Figura 3. Imagem macroscópica da peça cirúrgica, com visualização do tumor GIST aderido ao mesorreto. 


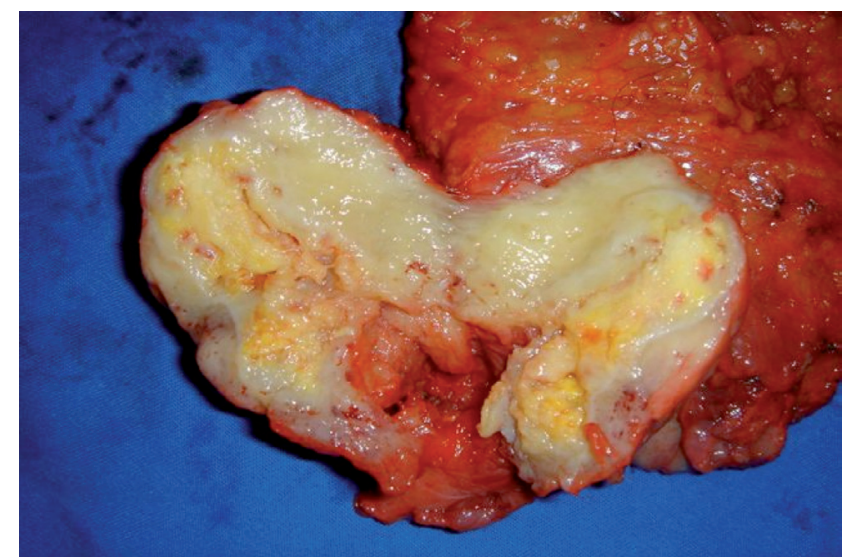

Figura 4. Peça cirúrgica com tumor GIST em destaque.

ressecção e colostomia ilíaca terminal. O estudo anatomopatológico com painel imunoistoquímico para GIST confirmou a natureza da lesão, que infiltrava toda a túnica muscular até a gordura mesorretal, sem metástases linfonodais. Após um ano, o paciente foi submetido ao fechamento da ileostomia com restabelecimento do trânsito pela colostomia, após recusa de nova tentativa de abaixamento coloanal. Encontra-se clinicamente muito bem, sem sinais clínicos de recorrência da doença, assim como à ressonância magnética da pelve.

\section{DISCUSSÃO}

A origem dos tumores derivados do GIST está relacionada às células intersticiais de Cajal (células marcapasso), que expressam normalmente o protoncogene $c$ $k i t^{1}$. Este protoncogene codifica um receptor para fator de crescimento com função tirosina-quinase, denominado receptor $c$-kit. São consideradas atualmente as neoplasias mesenquimais mais comuns do trato gastrintestinal. Em geral, acometem adultos acima dos 30 anos, a maioria dos tumores ocorre no estômago $(70 \%)$ e intestino delgado $(25 \%)^{2}$, sendo mais raros no intestino grosso, onde a maioria se localiza no reto $(5 \%)^{3}$. Os pacientes com GIST retal são predominantemente homens (71\%), entre 60 e 70 anos de idade ${ }^{3}$.

O tratamento do GIST se baseia na ressecção cirúrgica, com acompanhamento do paciente para rastreamento de metástases metacrônicas (fígado, peritônio, pulmões). Em seguimento médio de cinco anos, 64,3\% dos pacientes apresentam recorrência ou metástases à distância, o que acarreta sobrevida neste período menor que $50 \%{ }^{4}$.
A situação ideal para indicação de cirurgia é a presença de neoplasia primária, sem metástase, com possibilidade de ressecção completa (R0), o que frequentemente é possível, uma vez que tais neoplasias possuem pequena capacidade de infiltrar a parede das vísceras ${ }^{5}$. Desta forma, as margens de segurança não necessitam ser extensas. Admite-se que $4 \mathrm{~cm}$ de margem livre, as quais sejam avaliadas macroscopicamente, sejam suficientes. A disseminação linfonodal acontece raramente, não havendo necessidade de linfadenectomia extensa ${ }^{6}$. $\mathrm{O}$ tratamento adjuvante com quimioterapia ou radioterapia apresenta resultados desanimadores ${ }^{7}$.

Changchien et al. ${ }^{4}$ avaliaram 42 pacientes portadores de GIST retal, sendo que 29 foram submetidos à ressecção radical (amputação abdômino-perineal e ressecção anterior baixa) e 13, à ressecção local ampla (transretal e por via de Kraske). Dos pacientes submetidos à ressecção local, 77\% tiveram recorrência locorregional, enquanto daqueles submetidos à ressecção radical, 31\% recorreram localmente, com diferença estatisticamente significativa. Com relação a metástases à distância, não houve diferença estatisticamente significativa entre os dois grupos avaliados. Outros estudos sugerem que tratamentos mais agressivos e radicais devem ser indicados no tratamento dos GISTs retais. Pidhorecky et al. ${ }^{8}$ concluíram que os fatores mais importantes de pior prognóstico para a doença são: tamanho maior que $5 \mathrm{~cm}$, presença de necrose tumoral, infiltração profunda, metástases à distância e número de mitoses maior que cinco por dez campos microscópicos.

Diversos estudos têm demonstrado que os tumores GIST expressam o receptor tirosino-quinase, o qual é o produto do protoncogene $c$-kit $t^{6}$. A mutação do $c$-kit leva à ativação da tirosino-quinase, resultando em proliferação celular descontrolada e resistência à apoptose. O mesilato de imatinibe é um inibidor seletivo da tirosino-quinase, que bloqueia a atividade do $c$-kit nas células tumorais ${ }^{9}$. A efetividade do imatinibe no tratamento dos GISTs foi descrita inicialmente em 2001, o que resultou em dramática melhora dos pacientes com lesões metastáticas múltiplas 9 . Estudos de fase II levaram à sua aprovação em 2002, pela Agência Americana Reguladora de Medicamentos (FDA), para tumores metastáticos e nãoressecáveis, na dose de 400 a $600 \mathrm{mg}$ por dia. Observouse resposta parcial em 53,7\% e estabilidade da doença, em $27,9 \%$ dos casos, em 24 semanas de acompanhamento após o início da resposta ${ }^{7}$. O uso de imatinibe é bem 
tolerado de modo geral, com poucos efeitos colaterais, tais como: edema, diarreia e fadiga ${ }^{10}$.

Existem poucos relatos da utilização do mesilato de imatinibe no tratamento do GIST retal. Lo et al. ${ }^{10}$ relataram o caso de uma paciente com GIST retal e indicação de amputação abdômino-perineal, que foi recusada pela paciente, tendo sido a mesma submetida a tratamento com imatinibe. Após um mês de tratamento, houve diminuição da dor retal e melhora do calibre fecal, sendo que após seis semanas, a tomografia mostrou diminuição do tamanho do tumor. A paciente foi submetida à ressecção transanal, não tendo sido observada recorrência em 24 meses.

Hou et al. ${ }^{11}$, por sua vez, relataram o caso de um paciente com GIST retal, que foi submetido à neoadjuvância e depois à cirurgia. Este paciente estava assintomático e livre de doença 57 meses após o tratamento. Eles acreditam que esse resultado é em grande parte devido à neoadjuvância com imatinibe. Sendo assim, os autores consideram, com base neste caso e em outros relatados na literatura, que a neoadjuvância pode melhorar dramaticamente o prognóstico do GIST retal.

Também foi relatado por Hamada et al. ${ }^{12}$ o uso do imatinibe em um caso de terceira recorrência local de GIST retal. A cirurgia padrão para o caso deixaria o paciente com incontinência urinária e colostomia definitiva. Optou-se por realizar neoadjuvância com imatinibe, com redução importante do tamanho do tumor. Posteriormente, foi possível ressecar completamente o tumor com preservação das funções urinária e anal.

No presente caso, os efeitos colaterais da terapia neoadjuvante foram bem tolerados e o tumor diminuiu à metade, ao exame físico. A diminuição do volume da massa à ressonância, embora considerável, é difícil de ser quantificada, uma vez que a reação inflamatória peritumoral pós-imatinibe (flare) dificulta tal avaliação ${ }^{13}$.

Deve-se se salientar que o paciente seria inicialmente submetido à amputação abdômino-perineal, mas devido à resposta ao tratamento com imatinibe teve seu aparelho esfincteriano preservado, sendo possível a realização de retossigmoidectomia ultrabaixa com anastomose colo-anal, a princípio. Devido ao biótipo do paciente, o abaixamento do cólon resultou em sua isquemia, tendo sido necessária a ressecção do mesmo. Apesar desta evolução desfavorável, foi possível demonstrar a correção da conduta adotada no presente caso, podendo a mesma ser fortemente considerada em pacientes portadores de GIST volumosos de reto médio e inferior. Não foi indicado tratamento adjuvante com o imatinibe após a cirurgia, pois esta prática não é padrão para a doença não-metastática ${ }^{14}$.

\section{CONCLUSÃO}

O tratamento cirúrgico ideal para os GISTs de reto deve ser radical sempre que possível, a fim de diminuir as chances de recorrência local. Com o uso do mesilato de imatinibe e sua capacidade de regressão tumoral, lesões inicialmente irressecáveis ou passíveis de amputação retoanal podem ser tratadas com cirurgias radicais e não-mutiladoras. Entretanto, a escassez de dados na literatura demonstra que outros estudos são necessários para melhor definir o papel neoadjuvante do imatinibe no GIST retal, assim como a melhor tática de cirurgia a ser utilizada e o possível uso adjuvante da droga.

\footnotetext{
ABSTRACT: The role of drugs that intervene with the tirosine kinase activity on the c-kit receptors in the treatment of gastrointestinal stromal tumors (GISTs) has been considered very important, mainly in large tumors. We report a case of a male patient, 56 years-old, obese, presenting with feeling of rectal pressure and incomplete evacuation. Work-up revealed a large inferior rectal GIST located in the posterior wall, suspected on MRI and confirmed by immunohistochemical study of a parasacral biopsy guided by tomography. The supposed initial approach was an abdominoperineal resection, since tumor was compressing anal canal and sphincter complex. In order to save the sphincters, we have decided to refer patient to neoadjuvant treatment with imatinib mesylate (Glyvec ${ }^{\circledR}$ ). After four months of treatment, a down staging of tumor was observed during rectal exam (about 50\%), which was smaller on pelvic RNM. Patient was undergone to total mesorectal excision with manual coloanal anastomosis and protective ileostomy. He presented necrosis of mobilized left colon and underwent to resection, and terminal iliac colostomy. Subsequently, patient refused to undergo through a new coloanal anastomosis and remain with iliac colostomy after ileostomy takedown. In the treatment of unresectable or large rectal GISTs, the use of imantinib should be strongly considered, since that radical surgery is the main approach to reduce the possibility of local recurrence.
}

Keywords: rectal cancer; colorectal neoplasias; surgery; antineoplasic drugs. 


\section{REFERÊNCIAS}

1. Kindblom LG, Remotti HE, Aldenborg F, Meis-Kindblom JM. Gastro-intestinal pacemaker cell tumor (GI-PACT): gastrointestinal stromal tumors show phenotypic characteristics of the interstitial cells of Cajal. Am J Pathol. 1998;152:1259-69.

2. Miettinen M, Sarlomo-Rikala M, Lasota J. Gastrointestinal stromal tumours. Ann Chir Gynaecol. 1998;87:278-83.

3. Miettinen M, Furlong M, Sarlomo-Rikala M, Burke A, Sobin LH, Lasota J. Gastrointestinal stromal tumors, intramural leiomyomas, and leiomyosarcomas in the rectum and anus. Am J Surg Path. 2001;25:1121-33.

4. Changchien CR, Wu MC, Tasi WS, Tang R, Chiang JM, Chen JS, et al. Evaluation of prognosis for malignant rectal gastrointestinal stromal tumor by clinical parameters and immunohistochemical staining. Dis Colon Rectum. 2004;47(11):1922-9.

5. Connolly EM, Gaffney E, Reynolds JV. Gastrointestinal stromal tumours. Br J Surg. 2003;90(10):1178-86.

6. Sarlomo-Rikala M, Kovatich AJ, Barusevicius A, Miettinen M. CD117: a sensitive marker for gastrointestinal stromal tumors that is more specific than CD34. Mod Pathol. 1998;11(8):728-34.

7. Demetri GD, Mehren von M, Blanke CD, Van den Abbeele $\mathrm{AD}$, Eisenberg B, Roberts PJ, et al. Efficacy and safety of imatinib mesylate in advanced gastroin-testinal stromal tumors. N Engl J Med. 2002;347(7):472-80.

8. Pidhorecky I, CheneyRT, KraybillWG, Gibbs JF. Gastrointestinal stromal tumors: current diagnosis, biologic behavior, and management. Ann Surg Oncol. 2000;7(9):705-12.

9. Joensuu H, Roberts PJ, Sarlomo-Rikala M, Andersson LC, Tervahartiala P, Tuveson D, et al. Effect of the tyrosine kinase inhibitor STI571 in a patient with a metastatic gastrointestinal stromal tumor. N Engl J Med. 2001;344(14):1052-56.

10. Lo SS, Papachristou GI, Finkelstein SD, Conroy WP, Schraut WH, Ramanathan RK, et al. Neoadjuvant Imatinib in Gastrointestinal Stromal Tumor of the Rectum: Report of a Case. Dis Colon Rectum. 2005;48(6):1316-9.

11. Hou YY, Zhou Y, Lu SH, Hou J, Tan YS. Imatinib mesylate neoadjuvant treatment for rectal malignant gastrointestinal stromal tumor. World J Gastroenterol. 2009;15(15):1910-3.

12. Hamada M, Ozaki K, Horimi T, Tsuji A, Nasu Y, Iwata J, et al. Recurrent rectal GIST resected successfully after preoperative chemotherapy with imatinib mesylate. Int J Clin Oncol. 2008;13(4):355-60.

13. Stroszczynski C, Jost D, Reichardt P, Chmelik P, Gaffke G, Kretzschmar A, et al. Follow-up of gastro-intestinal stromal tumours (GIST) during treatment with imatinib mesylate by abdominal MRI. Eur Radiol. 2005;15(12):2448-56.

14. Wu PC, Langerman A, Ryan CW, Hart J, Swiger S, Posner MC. Surgical treatment of gastrointestinal stromal tumors in the imatinib (STI-571) era. Surgery. 2003;134(4):656-65.

\section{Endereço para correspondência:}

ANTÔNIO LACERDA FILHO

Clínica Coloproctológica - HFR

Avenida do Contorno, 9.530

CEP: 30110-934 - Belo Horizonte (MG), Brasil

E-mail: alacerdafilho@gmail.com 